\title{
ECOLOGÍA PARA LA CONSERVACIÓN DE LA DIVERSIDAD BIOLÓGICA
}

\author{
Javier A. Simonetti Zambelli
}




\section{JAVIER A. SIMONETTI ZAMBELLI}

Biólogo por la Universidad de Chile y Ph.D. por la Universidad de Washington. Profesor Titular de la Facultad de Ciencias, Universidad de Chile. Se ha interesado en la conservación de la diversidad biológica en Latinoamérica, particularmente desde la ecología, incorporando también los aspectos sociales asociados (www. conservacion.cl). Preside la Asociación Kauyeken, organización abocada a poner en valor la relación entre la naturaleza y la cultura nacional (www.kauyeken.cl). 


\section{ECOLOGÍA PARA LA CONSERVACIÓN DE LA DIVERSIDAD BIOLÓGICA}

\section{INTRODUCCIÓN}

La sociedad contemporánea enfrenta tiempos de cambios ambientales acelerados a escala planetaria. Resultado del tamaño y crecimiento de la población y el aumento en la demanda de recursos para satisfacer sus necesidades de bienes y servicios, numerosas actividades humanas tienen repercusiones sobre los componentes biofísicos de la naturaleza. Estos abarcan cambios en el patrón de uso de suelos, la circulación atmosférica y oceánica, los ciclos biogeoquímicos del carbono y del nitrógeno, el clima y la diversidad biológica, entre otros (Vitousek, 1994; Ecosystems Panel, 2000). Lo paradojal es que estos cambios afectan negativamente la calidad de vida de las generaciones actuales y comprometen aquella de las generaciones futuras (Norton, 1992).

La pérdida de diversidad biológica destaca entre estos cambios tanto por su irreversibilidad como por las consecuencias sobre la población humana. La extinción de genes y especies y ecosistemas conlleva la pérdida de procesos y linajes evolutivos, así como una alteración de la capacidad de los ecosistemas para proveer los bienes y servicios de los cuales la humanidad depende (Nee y May, 1997; Cardinale et al., 2012). Es decir, la pérdida de diversidad biológica constituye la pérdida de nuestro patrimonio natural.

Numerosas especies son valoradas por la población por motivos tan diversos como la propia valoración de su existencia, por poseer relevancia cultural y hasta por ser empleadas como recurso alimenticio, medicinal u otro. Al mismo tiempo, los procesos en que las especies participan, como el ciclado de nutrientes, también son valorados en tanto afectan la fertilidad del suelo y la productividad vegetal, entre otras condiciones ambientales que inciden directamente en el bienestar de las poblaciones humanas (Naeem et al., 2009).

Las causas proximales más importantes que inciden en el aumento de la tasa contemporánea de extinción son la pérdida de extensión y calidad del hábitat disponible para la biodiversidad (Sala et al., 2000). La reducción en cantidad y calidad de hábitat implica una reducción en la distribución y abundancia de las especies variables que inciden en el riesgo de extinción y son propias del ámbito conceptual de la ecología como ciencia biológica (Andrewartha y Birch, 1954). 
Históricamente, numerosas acciones se han emprendido para reducir la tasa a la cual se modifica la biodiversidad y para prevenir futuras extinciones producto de actividades antropogénicas (e.g., Taber y Payne 2003). La más común ha sido establecer áreas protegidas, tales como parques nacionales. El supuesto subyacente a esta aproximación es que segregando las especies y ecosistemas de las actividades humanas deletéreas se podría permitir que la biodiversidad subsistiera indefinidamente. Sin embargo, y pese a los esfuerzos por crear y mantener áreas protegidas, ellas por sí solas no serán suficientes para revertir la creciente tasa de extinciones. Las áreas protegidas han sido determinantes en reducir parcialmente la crisis de las extinciones de la biodiversidad, pero requieren de apoyo en dicha tarea (e.g., Pimm y Raven, 2000; Mora y Sale, 2011). De hecho, no obstante más de 20 millones de km2 están protegidos a nivel mundial, una fracción significativa de las especies conocidas no está resguardada en áreas protegidas y sobrevive en tierras destinadas a otros usos (Simonetti et al., 2013). Además, el estado de la biodiversidad sigue declinando. Un claro indicador es el Índice del Planeta Vivo, que evalúa el tamaño de 10.380 poblaciones de 3.038 especies de mamíferos, aves, reptiles, anfibios y peces en el mundo. Globalmente, este índice ha disminuido un $52 \%$ entre los años 1970 y 2010, sugiriendo que, en promedio, las poblaciones estudiadas se han reducido a la mitad en menos de medio siglo (McLellan, 2014).

El panorama actual sobre protección de la biodiversidad obliga no solamente a mejorar la cobertura y eficiencia de las áreas protegidas, sino a realizar gestiones para conservar la biodiversidad también fuera de las áreas protegidas. En efecto, para reducir las presiones sobre la biodiversidad, el Convenio sobre la Diversidad Biológica, del cual Chile es signatario, estableció como objetivo estratégico "abordar las causas subyacentes de la pérdida de diversidad biológica mediante la incorporación de la diversidad biológica en todos los ámbitos gubernamentales y de la sociedad", en cuyo marco fijó como meta que para el año 2020 "las zonas destinadas a agricultura, acuicultura y silvicultura se gestionarán de manera sostenible, garantizándose la conservación de la diversidad biológica" (CBD, 2010). Este requerimiento demanda mantener la producción de los bienes y servicios a los cuales dicha superficie se ha destinado junto con reducir los efectos negativos de los procesos productivos sobre la biodiversidad. Considerando que la satisfacción de los objetivos y metas establecidas por acuerdos internacionales, tales como el Convenio sobre la Diversidad Biológica, son trasladables a criterios de certificación de producción ambientalmente amigable, resulta crítico lograr establecer la forma en que dichos requerimientos pueden ser satisfechos (e.g., Auld et al., 2008). En esta nota presento un caso que establece que mediante la aplicación de conceptos básicos de ecología es factible mejorar las condiciones para la biodiversidad en plantaciones forestales, las que de adoptarse en la industria forestal lograrían 
satisfacer los compromisos contraídos respecto de conservar diversidad biológica en áreas destinadas a la producción de bienes forestales.

\section{BIODIVERSIDAD EN PLANTACIONES FORESTALES}

La demanda de productos como madera y celulosa ha implicado un constante aumento de las plantaciones forestales. Estas cubren aproximadamente más de 190 millones de hectáreas globalmente y se espera que crezcan a un ritmo anual de un 2\% (FAO, 2011). Estas plantaciones son generalmente consideradas como "desiertos biológicos" (Hartley, 2002). En general, cuando un bosque nativo es reemplazado por una plantación industrial, la riqueza y abundancia de las especies disminuye significativamente. Esto ocurre en grupos tan diversos como aves, mamíferos y anfibios. A nivel global, la riqueza de especies de mamíferos disminuye en un $81 \%$ de los casos en que un bosque es reemplazado por una plantación. Asimismo, las especies endémicas disminuyen en un $62 \%$ de los casos. Entre las aves, la riqueza disminuye en un $72 \%$ de las veces en que se reemplazan bosques por plantaciones forestales (Nájera y Simonetti, 2010a). Por su parte, entre los anfibios, la riqueza de especies disminuye en un $68 \%$ de los casos, en tanto entre los reptiles la riqueza de especies no varía significativamente entre los bosques originales y las plantaciones que los reemplazan. Sin embargo, la composición cambia, siendo dominada por especies generalistas de hábitat donde las especies amenazadas son menos frecuentes (Palacios et al., 2013).

En términos de abundancias poblacionales, las plantaciones forestales en general soportan una menor cantidad de individuos. Por ejemplo, la abundancia de los mamíferos disminuye en un $75 \%$ de los casos en que un bosque es reemplazado por una plantación, en contraste con la abundancia de aves y anfibios que no varía entre bosques y plantaciones y con la abundancia de reptiles, la que aumenta (Nájera y Simonetti, 2010a; Ramírez y Simonetti, 2011; Palacios et al., 2013).

Los cambios observados en riqueza y abundancia de especies ocurren independientemente del tipo de plantación que se trate y del bioma donde esta se desarrolle. La transformación de un bosque en plantación conlleva entonces la pérdida de hábitat y se traduce en una amenaza para la biota nativa. Por ejemplo, las actividades agroforestales son la amenaza más importante para las aves a nivel mundial. Un 93\% de las aves amenazadas de extinción está afectada por la pérdida y degradación de la calidad de su hábitat, la cual está asociada a actividades agrícolas y forestales (Vié et al. 2009).

En Chile, las plantaciones de pino Monterrey (Pinus radiata) cubren sobre 1,5 millones de hectáreas y se encuentran establecidas en la región central de Chile, zona caracterizada por su elevado endemismo, por lo que impactan sobre una 
diversidad biológica única y de relevancia mundial. Al igual que lo que ocurre a nivel mundial, estas plantaciones modifican significativamente la biodiversidad (véase Estades et al., 2012 para una revisión detallada). Por ejemplo, la riqueza de especies de coleópteros epigeos es menor, pero su abundancia no varía entre los bosques nativos y las plantaciones, en contraste con la riqueza de aves, que no difiere, y las abundancias totales son mayores en las plantaciones (Estades y Temple, 1999; Grez et al., 2003). Sin embargo, la riqueza y abundancia varía según los hábitos tróficos de las aves, donde aves insectívoras que forrajean en el suelo pueden ser más comunes en plantaciones, pero las insectívoras de follaje son menos frecuentes (Vergara y Simonetti, 2004). Las plantaciones contienen además un mayor número de especies exóticas que los bosques. En efecto, la riqueza de especies de plantas, insectos, aves y mamíferos exóticos es mayor en plantaciones, las que podrían actuar como un foco de dispersión hacia el bosque. Por ejemplo, el coleóptero Hylaster ater, plaga de las plantaciones de pino, ha comenzado a invadir la vegetación nativa (Grez et al., 2003). Así, las plantaciones representan otra amenaza a la biodiversidad nativa (Becerra y Simonetti, 2013).

Por lo tanto, a nivel global y nacional, las plantaciones forestales efectivamente constituyen una amenaza a la biodiversidad, la cual no solo debe ser reducida sino que debe transformarse en colaboradora en mantener la biodiversidad, acorde con los requisitos del Convenio sobre Diversidad Biológica (CBD, 2010).

\section{UN PAR DE APORTES DESDE LA ECOLOGÍA}

La meta del Plan Estratégico del Convenio sobre la Diversidad Biológica respecto de lograr que las áreas abocadas a producción de bienes, como los forestales, se gestionen de manera de garantizar la conservación de la diversidad biológica (CBD, 2010), implica transformar una amenaza en una oportunidad de colaborar con su mantención.

Un factor ambiental que contribuiría a la disminución de las especies nativas es la simplificación estructural de las plantaciones. Manejadas de manera de simplificar su estructura y estandarizarlas, la remoción de elementos como troncos y ramas caídas y vegetación del sotobosque reduce la heterogeneidad ambiental (Puettmann et al., 2009). Reducir la complejidad estructural implicaría disminuir el espectro de recursos disponibles y con ello, reducir la cantidad de especies que la plantación forestal, en tanto hábitat, sería capaz de sostener (cf. MacArthur, 1972). En este contexto, una forma de reducir el impacto sobre la biodiversidad, en término de riqueza y abundancia de especies, sería aumentar la complejidad estructural de las plantaciones (e.g., Grez et al., 2003, Lindenmayer and Hobbs 2004). 
La reducción en riqueza y abundancia de especies en plantaciones forestales comparadas con el bosque original no ocurre en todos y cada uno de los casos. De hecho, hay ocasiones en las cuales estos factores no varían. Un análisis de los casos en los cuales la riqueza no disminuye revela que las plantaciones exhiben un sotobosque desarrollado, vegetación que ofrecería una mayor cantidad de recursos, tales como hábitat y alimento a las especies nativas (Ramírez y Simonetti, 2011). De hecho, si se comparan plantaciones "clásicas", sin sotobosque en su interior, con plantaciones con desarrollo de vegetación en el sotobosque, la riqueza de especies y la abundancia de aves son mayores en plantaciones con sotobosque en el $79 \%$ y $92 \%$ de los casos, respectivamente (Nájera y Simonetti, 2010a). De igual forma, en un $90 \%$ de los casos la riqueza de especies y en un $75 \%$ de las especies su abundancia es mayor en plantaciones estructuralmente complejas al contener un sotobosque desarrollado (Ramírez y Simonetti, 2011). Por tanto, la existencia de mayor complejidad estructural mitigaría los efectos negativos de las plantaciones sobre la biota nativa.

La propuesta de que al aumentar la complejidad estructural se amplía el espectro de recursos potenciales para la biota nativa y con ello se facilita el uso de las plantaciones como hábitat requiere ser demostrada experimentalmente a lo menos por dos motivos. Primero, la asociación entre mayor riqueza de especies y presencia de sotobosque es correlacional y no una demostración empírica de causalidad. Esta demostración es necesaria por cuanto el sotobosque es usualmente eliminado o inhibido en las plantaciones tradicionales. El segundo motivo entonces radica en que la presencia de un sotobosque desarrollado atentaría contra las prácticas clásicas, entre cuyos supuestos se sostiene que toda vegetación en el sotobosque competiría por recursos con los árboles plantados, lo cual reduciría su crecimiento y, por tanto, la rentabilidad (Puettmann et al., 2009). Esto haría poco factible la incorporación de esta sugerencia en los planes de manejo de las plantaciones y con ello avanzar en el cumplimiento de los compromisos en conservación de biodiversidad (cf. CBD, 2010)

Respecto a la relación entre sotobosque y riqueza de especies, dos experimentos manipulativos en condiciones de campo (sensu Diamond, 1986) han demostrado inequívocamente que las plantaciones con un sotobosque desarrollado sostienen más especies que aquellas sin sotobosque. Si la presencia de un sotobosque desarrollado fuese, como sugiere la evidencia disponible, el factor que favorece la presencia de la fauna en las plantaciones, su remoción debería generar reducciones en riqueza y en abundancia, al punto que las plantaciones donde experimentalmente se ha removido el sotobosque deberían ser similares en riqueza y abundancia a plantaciones que no soportan vegetación bajo el dosel. En efecto, al remover experimentalmente el 
sotobosque desde rodales en plantaciones de palma aceitera (Elaeis guineensis) en Guatemala, como en plantaciones de pino Monterrey (P. radiata) en Chile, tanto la riqueza como la abundancia de especies disminuye en comparación con la riqueza y abundancia en las mismas antes que el sotobosque fuese retirado para simular plantaciones sin vegetación (Nájera y Simonetti, 2010b; Simonetti et al., 2013, Cerda et al. 2015). En las plantaciones de palma aceitera la riqueza de especies de aves se redujo en un 41\%, en tanto la abundancia decrece en un 60\% (Nájera y Simonetti. 2010b). En plantaciones de pino la riqueza de especies de mamíferos de talla mediana, incluyendo al zorro culpeo (Pseudalopex culpaeus), la güiña (Leopardus guigna), el chingue (Conepatus chinga) y el pudú (Pudu puda), se reduce de cuatro a una especie, cuya abundancia baja 2,5 veces respecto de la situación con sotobosque (Simonetti et al., 2013). Asimismo, al removerse el sotobosque, la abundancia del peorro (Ceroglossus chilensis) en las plantaciones que contenían vegetación bajo el dosel se reduce hasta valores equivalentes a la abundancia de peorros en plantaciones sin sotobosque (Cerda et al., 12015). Independiente de si la vegetación del sotobosque ofrece refugio, alimento u otros recursos que inciden en su calidad de hábitat, estos resultados demuestran que su presencia-cualquiera sea la causa más próxima- mejora su condición para la fauna, reduciendo el síndrome del "desierto biológico", proveyéndose entonces de una dimensión ambiental manejable para lograr conservar biodiversidad en plantaciones forestales.

Para que el manejo de las plantaciones considere favorecer el desarrollo del sotobosque, la presencia del mismo debería ser neutra respecto al objetivo productivo perseguido por las plantaciones. Si bien en el estado de plántulas la vegetación acompañante reduce el crecimiento de los pinos al competir por agua, luz y nutrientes (Lewis et al., 1993), ello no implica necesariamente que la competencia persista en estados mayores, cuando el dosel de las plantaciones se cierra. De hecho, el crecimiento medio anual de los pinos no se correlaciona con el volumen de la vegetación que rodea a cada árbol dentro de un rodal. Asimismo, al removerse experimentalmente esta vegetación, el crecimiento medio anual no se incrementa en comparación con su crecimiento previo ni con aquellos árboles creciendo en plantaciones sin sotobosque (Simonetti et al., in letteris). De existir competencia, al removerse el sotobosque el crecimiento debería aumentar, pues se habrían liberado los recursos por los cuales compiten. Ello no ocurre, pudiendo descartarse interacciones competitivas entre el sotobosque y las plantaciones adultas de pino, lo que elimina el problema potencial de una reducción en productividad forestal. Es más, la presencia de sotobosque y con ello, de fauna, podría ser beneficiosa para las plantaciones al soportar una fauna de aves insectívoras que podrían reducir la abundancia o los efectos de insectos que pudiesen afectar el crecimiento de los árboles. 
Un experimento natural, contrastando el riesgo de depredación de larvas de insectos y la magnitud de la herbivoría en plantaciones con niveles contrastantes de sotobosque, revela que una larva de insecto tiene una probabilidad dos veces más alta de ser atacada en una plantación estructuralmente compleja que en una simple, sin sotobosque, en tanto la magnitud de la herbivoría es significativamente menor en las plantaciones con sotobosque desarrollado. La mayor parte de las larvas son atacadas por aves (85\% de los ataques) y secundariamente por insectos $(15 \%$; Poch y Simonetti, 2013). La reducción en herbivoría ocurre en las plantaciones de forma similar a lo que acontece en los bosques nativos, donde la presencia de aves también reduce significativamente la herbivoría (De la Vega et al., 2013). Es decir, la presencia de aves en las plantaciones con sotobosque se traduce en un servicio ecosistémico potencial - control de plagas-, generándose un escenario "ganar-ganar", donde las superficies destinadas a plantaciones dejan de actuar como desiertos biológicos y se transforman en hábitat alternativos para la biodiversidad, en tanto las plantaciones ganan un controlador de plagas (Poch \& Simonetti, 2013).

Las plantaciones con sotobosque desarrollado también constituyen un hábitat para diferentes especies de murciélagos insectívoros. Tanto el murciélago rojo (Lasiurus varius) como el murciélago oreja de ratón (Myotis chiloensis) son más abundantes en plantaciones de pino con sotobosque que en plantaciones desprovistas de esta vegetación, los que se desplazan por las plantaciones a través de los caminos de servicios forestales. Además, todas las especies de murciélagos son muy activas en los bordes de las plantaciones, lo cual aumenta la probabilidad de que las especies nativas presenten servicios de control de plaga en las plantaciones forestales (Rodríguez-San Pedro y Simonetti, 2103, 2015).

De esta forma, si se permite o facilita el desarrollo de vegetación bajo el dosel de las plantaciones de pino se pueden minimizar sus impactos sobre la riqueza y abundancia de especies y de las interacciones en que ellas participan. Estos hechos refuerzan la posibilidad de que las plantaciones pudiesen ser manejadas como hábitat alternativo para la fauna nativa, acorde con los requerimientos contraídos en el Convenio sobre la Diversidad Biológica, junto con orientarse a la producción forestal.

\section{ECOLOGÍA Y ALGO MÁS}

Descansando en el “cuadrante Pasteur" (Stokes, 1997), donde se combina un marco conceptual desde la teoría ecológica (teoría del nicho y el papel de la heterogeneidad en la estructuración comunitaria, junto con la teoría de la competencia) con una necesidad social concreta (reducir las presiones sobre la biodiversidad), es factible 
proponer aproximaciones que incrementen la sustentabilidad de una actividad productiva y de esta forma abordar cabalmente el problema de la gestión de la biodiversidad como un problema científico de relevancia social (Simonetti, 2011). Ello es más urgente en tanto la demanda por productos forestales continuará aumentando en los años venideros. El incremento en la población humana y sus requerimientos de productos forestales y de energía podrían triplicar las demandas sobre los bosques y las plantaciones hacia el año 2050 (WWF 2015).

No obstante el objetivo primario de las plantaciones es la producción de madera, celulosa y otros productos forestales, se espera que colaboren de manera efectiva en la conservación de la biodiversidad -opuesto a lo que ha sido su legado-. La evidencia empírica demuestra que ello es factible en tanto el manejo de las plantaciones permite o facilita el desarrollo de una vegetación bajo el dosel (Simonetti et al., 2012 para revisiones en diferentes tipos de plantaciones en el mundo). Aproximaciones como esta contribuirían a recudir las presiones sobre las especies que sobreviven fuera de las áreas protegidas (e.g., Pimentel et al., 2002).

Numerosas especies de plantas vasculares, la mayoría nativas, crecen bajo el dosel de las plantaciones de pino en Chile, (e.g., Ramírez et al., 1984; Poch y Simonetti, 2013), las cuales pueden contribuir a mejorar la calidad de las plantaciones como hábitat, de forma que una práctica sencilla sería no interferir con el desarrollo de esta vegetación. Atendido que la evidencia sugiere que en estados adultos esta vegetación no compite con los pinos y por tanto no reduce su productividad, no existirían motivos para no adoptar esta práctica.

Además de los argumentos provenientes desde la ecología, la adopción de prácticas amigables con la biodiversidad es favorecida por la sociedad civil. Por una parte, en términos de preferencias por paisajes, la población prefiere las plantaciones con un sotobosque desarrollado en comparación con una plantación tradicional, sin sotobosque. Su nivel de preferencia es similar al mostrado por la población hacia un bosque nativo, como el bosque maulino (Püschel-Hoeneisen y Simonetti, 2012). Por otra, aun cuando las personas puedan no considerar las plantaciones de pino como una amenaza a la biodiversidad, en general ellas manifiestan preferir aquellas plantaciones que sirvan de hábitat para la fauna en peligro de extinción y estarían disponibles para pagar precios más altos por productos forestales que contribuyan a la conservación de biodiversidad en las plantaciones forestales. Estos resultados sugieren que las medidas destinadas a la conservación en las plantaciones forestales podrían ser apoyadas por la sociedad chilena (Püschel-Hoeneisen y Simonetti, 2012; véase también Zorondo-Rodríguez et al., 2014).

La globalización de la economía chilena y una creciente demanda nacional por productos ambientalmente amigables requiere de los productores la adopción genuina de prácticas que satisfagan estos crecientes estándares de calidad (Figueroa y 
Simonetti, 2006). Abandonar su calidad de "desiertos biológicos" sería un sustancial avance en el aporte de las plantaciones forestales a la conservación (CBD, 2010). Pese a que la biodiversidad se menciona explícitamente como un componente de la certificación forestal (e.g. CERTFOR, 2007), esta no se incorpora a las plantaciones mismas, sino que se espera sea resguardada en remanentes de vegetación nativa que no serían ni reemplazados por plantaciones ni intervenidos durante las faenas forestales. La evidencia proveniente desde la ecología informa claramente el conjunto de condiciones bajo las cuales las plantaciones pueden contribuir a la conservación integrando la fauna nativa, no segregándola, ofreciendo una aproximación que podría ser trasladada a la política pública en los estándares de certificación.

La segregación de la biota dentro de las áreas destinadas a plantaciones forestales emula a menor escala la aproximación empleada para establecer áreas protegidas, suponiendo que la segregación es necesaria y suficiente para resguardar la biota e ignorando el valor potencial de las plantaciones en sí mismas como hábitat alternativo (e.g., Fisher et al.,2008). Si las plantaciones chilenas u otras fuesen manejadas para proveer hábitat para la biota nativa y certificadas por ello, se avanzaría en desarrollar una industria más sustentable, como lo demanda la sociedad contemporánea, con una pequeña ayuda desde la ecología.

\section{AGRADECIMIENTOS}

Nuestro trabajo en conservación de biodiversidad en ambientes productivos se ha beneficiado de la colaboración de A.A. Grez, C.F. Estades, F. Zorondo, de nuestros estudiantes de pre y posgrado y del incansable apoyo de R. Zuñiga. Estas investigaciones han sido financiadas por FONDECYT (proyectos 1095046 y 1140657) y el Programa Domeyko-Biodiversidad, Universidad de Chile.

\section{REFERENCIAS}

Andrewartha, H.G., Birch, L.C. The distribution and abundance of animals. Chicago, University of Chicago Press, 1954, 782 págs.

Auld, G., Gulbrandsen, L.H., McDermott, C.L. "Certification schemes and the impacts on forests and forestry", en Annual Review of Environment and Resources, 2008, Vol. 33, pp. 187-211.

Becerra, P.I., Simonetti, J.A. "Patterns of exotic species richness of different taxonomic groups in a fragmented landscape of central Chile", en Bosque, Vol. 34, 2013, pp. 45-51. 
Cardinale, B.J., Duffy, J.E., Gonzalez, A., Hooper, D.U., Perrings, C., et al. "Biodiversity loss and its impact on Humanity", en Nature, 2012, Vol. 486, pp. 59-67.

CBD (Convention on Biological Diversity). Strategic plan for biodiversity 2011-2020 and the Aichi targets. Montreal, Secretariat of the Convention on Biological Diversity, 2010, disponible en http://www.cbd.int/sp/targets.

Cerda, Y., Grez, A.A., Simonetti, J.A. "The role of the understory on the abundance, movement and survival of Ceroglossus chilensis in pine plantations: an experimental test", en Journal of Insect Conservation, 2015, Vol. 19, pp. 119-127.

CERTOR (Sistema Chileno de Certificación de Manejo Forestal Sustentable). Estándar CERTFOR de manejo forestal sustentable para plantaciones. Santiago, Certfor, 28 págs.

De la Vega, X., Grez, A.A., Simonetti, J.A. "Is top-down control by predators driving insect abundance and herbivory rates in fragmented forests?" en Austral Ecology, 2012, Vol. 37, pp. 836-844.

Diamond, J. M. 1986. "Overview: laboratory experiments, field experiments, and natural experiments", en Diamond, J, Case, T.J. (editores). Community Ecology, New York: Harper and Row, 1986, pp. 3-22.

Ecosystems Panel, National Research Council. Global changes ecosystems research. Washington, D.C., National Academies Press, 2000, 60 págs.

Estades, C.F., Temple, S.A. "Deciduous-forest bird communities in a fragmented landscape dominated by exotic pine plantations", en Ecological Applications, 1999, Vol. 9, pp. 573-585.

Estades, C.F., Grez, A.A., Simonetti, J.A. "Biodiversity in Monterrey pine plantations, en Simonetti, J.A., Grez, A.A., Estades, C.F. (editores). Biodiversity conservation in agroforestry landscapes: challenges and opportunitites. Santiago, Editorial Universitaria, 2012, pp. 77-98.

FAO (Food and Agriculture Organization of the United Nations). State of the world's forests 2011. Roma, FAO, 2011, 164 págs.

Figueroa, E., Simonetti, J.A. (editores). Globalización y biodiversidad: oportunidades y desafíos para la sociedad chilena. Santiago, Editorial Universitaria, 2006, 328 págs.

Fischer, J., Brosi,B., Daily, G.C., Ehrlich, P.R. Goldman, R., et al. "Should agricultural policies encourage land sparing or wildlife friendly farming?" en Frontiers in Ecology and the Environment, 2008, Vol. 6, pp. 380-385. 
Grez, A.A., Moreno, P., Elgueta, M. “Coleópteros (Insecta: Coleoptera) epigeos asociados al bosque Maulino y plantaciones de pino aledañas", en Revista Chilena de Entomología, 2003, Vol. 29, pp. 9-18.

Hartley, M. "Rationale and methods for conserving biodiversity in plantation forests", en Forest Ecology and Management, 2002, Vol. 155, pp.81-95.

Lewis, N.B., Ferguson, I.S.; Sutton, W.R.J., Donald, D.G.M., Lisboa, H.B. Management of radiata pine. Butterworth-Heinemann, Inkata Press, 1993, 404 págs.

Lindenmayer, D.B., Hobbs, R.J. "Fauna conservation in Australian plantation forests - a review", en Biological Conservation, Vol. 119, 2004, pp. 151-18.

MacArthur, R.H. Geographical ecology. Patterns in the distribution of species. New York, Harper \& Row Publishers, 1972, 269 págs.

McLellan, R. (editor). Living Planet Report 2014. Species and spaces, people and places. Zurich, WWF International, 2014, 180 págs.

Mora, C., Sale, P.F. "Ongoing global biodiversity loss and the need to move beyond protected areas: a review of the technical and practical shortcomings of protected areas on land and sea”, en Marine Ecology Progress Series, 2011, Vol. 434, pp. 251-266.

Naeem, S., Bunker, D.E., Hector, A., Loreau, M., Perrings, C. Biodiversity, ecosystem functioning, and buman wellbeing. An ecological and economic perspective. Oxford, Oxford University Press, 2009, 368 págs.

Nájera, A., Simonetti, J.A. "Enhancing avifauna in commercial plantations", en Conservation Biology, 2010a, Vol. 24, pp. 319-324.

Nájera, A., Simonetti,J.A. “Can oil palm plantations becomebird friendly?” Agroforestry Systems, 2010b, Vol. 80, pp. 203-209.

Nee, S., May, R.M. "Extinction and the loss of evolutionary history", en Science, 1997, Vol. 278, pp. 692-694.

Norton, B. "Sustainability, human welfare and ecosystem health", en Environmental Values, 1992, Vol. 1, pp. 97-111.

Palacios, C.P., Agüero, B., Simonetti, J.A. "Agroforestry systems as habitat for herpetofauna: is there supporting evidence?" en Agroforestry Systems, 2013, Vol. 87, pp. 517-523.

Pimentel, D., Stachow, U., Takacs, D.A., Brubaker,H.W., Dumas, A.R. et al. "Conserving biological diversity in agricultural/forestry systems", en BioScience, 1992, Vol. 42, pp. 354-362 
Pimm, S.L., Raven, P. “Extinction by numbers”, en Nature, 2000, Vol. 403, pp. 843845.

Poch, T.J., Simonetti, J. A. "Insectivory in Pinus radiata plantations with different degree of structural complexity", en Forest Ecology and Management, 2013, Vol. 304, pp.132-136.

Puettmann, K.J., Coates, K.D., Messier, C. A critique to silviculture. Managing for complexity. Washington D.C., Island Press, 2009, 188 págs.

Püschel-Hoeneisen, N., Simonetti, J.A. "Forested habitat preferences by Chilean citizens: implications for biodiversity conservation in Pinus radiata plantations". Revista Chilena de Historia Natural, 2012, Vol. 85, pp.161-169.

Ramírez, C., Figueroa, H., Carrillo, R., Contreras, D. "Estudio fitosociológico de los estratos inferiores en un bosque de pino (Valdivia, Chile)", en Bosque, 1994, Vol. 5, pp. 65-81.

Ramírez, P. Simonetti, J.A. "Conservation opportunities in commercial plantations: the case of mammals", en Journal for Nature Conservation, 2011, Vol. 19, pp. 351-355.

Rodríguez-San Pedro, A., Simonetti, J.A. "Foraging activity by bats in a fragmented landscape dominated by exotic pine plantations in central Chile", en Acta Chiropterologica, 2013, Vol. 15, pp. 393-398

Rodríguez-San Pedro, A., Simonetti, J.A. "Does understory clutter reduce bat activity in forestry pine plantations?" en European Journal of Wildlife Research, 2015, Vol. 61, pp. 177-179

Sala, O.E., Chapin, F.S., Armesto, J.J., Berlow, E., Bloomfield, J., Dirzo, R., et al. "Global biodiversity scenarios for the year 2100", en Science, 2000, Vol. 287, pp. $1770-17$

Simonetti, J.A. "Conservation biology in Chile: Are we fulfilling our social contract? en Revista Chilena de Historia Natural, 2011, Vol. 84, pp. 161-170.

Simonetti, J.A., Grez, A.A., Estades, C.F. Biodiversity conservation in agroforestry landscapes: challenges and opportunities. Santiago, Editorial Universitaria, 2012, 162 págs.

Simonetti, J.A., Grez, A.A., Estades, C.F. "Providing habitat for native mammals through understory enhancement in forestry plantations", en Conservation Biology, 2013, Vol. 27, pp. 1117-1121.

Simonetti, J.A., Estades, C.F. Pérez, F.M. Grez A.A. "Does the understory compete with adult Monterey pines (Pinus radiata): an experimental test". In letteris. 
Stokes, D.E. Pasteur's quadrant: basic science and technological innovation. Washington, Brookings Institution Press, 1997, 180 págs.

Taber, R.D., Payne, N.F. Wildlife, conservation and human welfare. Malabar, Krieger Publishing Company, 2003, 218 págs.

Vergara, P.M., Simonetti, J.A. "Avian responses to fragmentation of the Maulino forest in central Chile", en Oryx, 2004, Vol. 38, pp. 383-388.

Vié, J.C., Hilton-Taylor, C., Stuart, S.N. (editores) (2009). Wildlife in a changing world. An analysis of the 2008 IUCN Red List of Threatened Species. Gland, International Union for Conservation of Nature, 2009, 180 págs.

Vitousek, P.M. "Beyond global warming: ecology and global change", en Ecology, 1994, Vol. 75, pp. 1861-1876.

WWF (Word Wide Fund for Nature). Living Forests Report. Gland, WWF International, 2015, 52 págs.

Zorondo-Rodríguez, F., Reyes-García, V., Simonetti, J.A. "Conservation of biodiversity in private lands: are Chilean landowners willing to keep threatened species in their lands?”, en Revista Chilena de Historia Natural, 2014, Vol. 87, disponible en http://www.revchilhistnat.com/content/1/1/4 